\title{
Mortalidad en pacientes menores de edad con diagnóstico de dengue y su relación con el uso de Dipirona
}

\author{
Mortality in minor patients with diagnosis of dengue and its relationship with the use of Dipirone
}

\author{
Oscar Alexander Gutiérrez-Lesmes ${ }^{*}$, Laura Inés Plata-Casas², Sandra Carolina Montaño-Contreras ${ }^{3}$
}

1 Enfermero, Esp. en Epidemiología, Magister en Gestión Ambiental Sostenible. Profesor Escuela de Salud Pública, Universidad de los Llanos. Villavicencio, Colombia. e-mail: oagutierrez@unillanos.edu.co ORCID: http://orcid.org/0000-0002-5181-0236

2 Bacterióloga, Esp. en Epidemiología, Magister en Salud Ocupacional. Profesora Escuela de Salud Pública, Universidad de los Llanos. Villavicencio, Colombia. e-mail: Iplata@unillanos.edu.co ORCID: http://orcid.org/0000-0002-0375-8875

3 Bacterióloga, Esp. en Epidemiología, Profesora Escuela de Salud Pública, Universidad de los Llanos. Villavicencio, Colombia. e-mail: smontano@unillanos.edu.co ORCID: http://orcid.org/0000-0003-0789-9525

\section{Resumen}

Introducción: El dengue es una de las diez causas de hospitalización más frecuentes en los servicios de urgencias de pediatría, en la atención sintomática se utiliza la Dipirona como antipirético, la guía clínica de manejo del dengue de la Organización Panamericana de la Salud (OPS) evidencia restricciones para su uso en pacientes con dengue y según la Guía de Atención Clínica Integral del paciente con dengue, no se debe administrar en pacientes pediátricos. Objetivo: Determinar la relación de la Dipirona con casos de mortalidad en menores de 16 años con dengue en el Meta. Materiales y métodos: Se realizó un estudio de casos y controles basado en la mortalidad por dengue en menores de edad reportados al Sistema de Vigilancia en Salud Pública del departamento del Meta, años 2008 a 2011. Resultados: La razón de las ventajas (OR) evidencia que la mortalidad por dengue es 17,6 veces mayor en menores de edad que recibieron Dipirona con un intervalo de confianza de 95\% entre 3,7-84,5 y un valor de P de 0,0002. Conclusión: El grupo de casos que recibió Dipirona en su tratamiento presentó una mayor mortalidad, la revisión de literatura no evidencia resultados sobre la relación de la Dipirona y mortalidad en niños con dengue, se debe seguir estudiando el fenómeno para contar con mayor evidencia científica.

Palabras clave: Dipirona; dengue; casos y controles; mortalidad. (Fuente: DeCS, Bireme).

\begin{abstract}
Introduction: Dengue is one of the ten most frequent causes of hospitalization in the emergency department of pediatrics where Dipyrone is used as an antipyretic in the symptomatic care. The clinical management guide for dengue of the Panamerican Health Organization (PHO) evidences restrictions for its use in patients with dengue, and according to the Integral Clinical Care Guide for patients with dengue, it should not be administered in pediatric patients. Objective: To determine the relationship between the uses of Dipyrone with the cases of mortality in children under 16 who suffered from dengue in Meta. Materials and methods: A case-control study was conducted based on mortality from dengue in minors reported to the Surveillance System in Public Health in the department of Meta, from 2008 to 2011. Results: The odds ratio (OR) shows that dengue mortality is 17.6 times greater in children Dipyrone
\end{abstract}


treated with a confidence interval of $95 \%$ between 3.7 to 84.5 and a P value of 0.0002 . Conclusion: The case group who received Dipyrone in their treatment had a higher mortality. There is no evidence or results in the literature review about the relationship of Dipyrone and mortality in children with dengue. This phenomenon should continue to be studied to have greater scientific evidence.

Keywords: Dipyrone; dengue; cases and controls; mortality. (Source: DeCS, Bireme).

\section{Introducción}

El dengue es una enfermedad causada por el virus del dengue (Arbovirus de la familia Flaviviradae), transmitida por el mosquito Aedes aegypti. Se manifiesta como enfermedad febril, acompañada de dolor (muscular, óseo, retrocular) y exantema ${ }^{1}$.

En países del centro y de Suramérica donde el dengue ha sido una enfermedad endémica, se ha reportado un patrón cambiante en la población a riesgo de padecer dengue siendo los menores de edad los más afectados². En las Américas la incidencia de dengue grave tiene un comportamiento poblacional que afecta a niños menores de 1 año de edad, escolares entre 5 a 9 años y adultos jóvenes ${ }^{3-5}$.

El dengue es una de las diez causas de hospitalización más frecuentes en los servicios de urgencias de pediatría, y casi un 25\% de los consultantes tienen enfermedad severa ${ }^{6,7}$. El mayor impacto se observa en los lactantes de siete meses en promedio, en los cuales se asocia al antecedente materno de infección reciente o al traspaso de anticuerpos tipo IgM a través de la placenta; y niños de 5 a 9 años a infección previa que indujo inmunidad heteróloga no protectora ${ }^{8}$.

El dengue en niños y jóvenes es particularmente riesgoso debido a las características clínicas y complicaciones tempranas asociadas con un curso rápido y fulminante con la afectación de órganos, como el miocardio e hígado, que llevan a una evolución fatal2.

El dengue es una de las principales causas de hospitalización y muerte en los países de latinoamérica ${ }^{9}$, la tasa de mortalidad calculada para dengue hemorrágico en la mayoría de países está alrededor del 5\%; siendo los niños y adultos jóvenes mayormente afectados ${ }^{10}$. Entre los factores que se han asociado con la mortalidad por dengue se han identificado los que facilitan la transmisión del virus, la severidad de la enfermedad, las barreras de acceso a los servicios de salud, el residir en municipios con una población menor de 100,000 habitantes, edad mayor a 65 años ${ }^{11}$; esta mortalidad debida a dengue se ha considerado como evitable y su reducción depende de la identificación oportuna de los casos, el acceso adecuado a la atención en los servicios de salud y la calidad de la atención que se proporcione a las personas afectadas ${ }^{12}$. La mortalidad en Colombia por dengue es mayor en niños de 1 a 5 años, en comparación con los grupos de lactantes menores de un año y niños mayores de 5 años ${ }^{13}$. El departamento del Meta presenta históricamente casos de mortalidad y morbilidad por dengue ${ }^{14}$.

La mortalidad por dengue se ha descrito como causa el choque hipovolémico y en la actualidad, se considera el compromiso de órganos como el hígado y el miocardio, como contribuyentes a la evolución fatal ${ }^{13,15}$.

Es en la atención de la enfermedad febril y el control del dolor ocasionados por el dengue que se utilizan los antinflamatorios no esteroideos (AINES); entre estos la Dipirona, también conocida como Metamizol perteneciente a la familia de las Pirazolonas, es un analgésico, antipirético, espasmolítico y antiinflamatorio, debido a la inhibición de la síntesis de prostaglandinas y al bloqueo de receptores del dolor a nivel central y periférico ${ }^{16,17}$, cuenta con un amplio uso en Colombia y latinoamérica ${ }^{18}$.

\section{Materiales y métodos}

Se realizó un estudio de casos y controles basado en la mortalidad por dengue en menores de edad reportados al Sistema de Vigilancia en Salud Pública del departamento del Meta, durante los 
años 2008 a 2011. Se determinó como caso todos los menores de edad con diagnóstico de dengue que fallecieron en estos años en el departamento del Meta y como controles a los menores de edad diagnosticados con dengue que no fallecieron, los controles fueron seleccionados al azar de las bases de datos de los mismos años de los casos; el grupo de casos estuvo constituido por 14 individuos menores de 16 años, el grupo control estuvo conformado por 56 individuos menores de 16 años, la relación caso control fue de 1:4.

Se revisaron las historias clínicas de los casos y los controles en las instituciones de primer y segundo nivel donde recibieron atención clínica por el dengue, verificando el tratamiento farmacológico recibido para fiebre y dolor, se definió como exposición el recibir Dipirona al menos una aplicación durante el tratamiento de la sintomatología por dengue. Se realizó análisis de razón de ventaja (OR), sus intervalos de confianza y prueba exacta de Fisher debido al número de unidades de análisis en SPSS versión 23.

\section{Consideraciones éticas}

La investigación no tuvo contacto con los sujetos de estudio, ya que el método de recolección fue documental, no se realizó ninguna intervención o modificación intencionada en los sujetos de estudio por lo anterior esta Investigación se clasifica sin riesgo según los parámetros de la
Resolución No. 8430 de 1993 del Ministerio de Salud.

\section{Resultados}

En la tabla 1 se observa el promedio de edad de los grupos, la comparación del promedio de edad para las medias no evidenció diferencia estadística significativa, (ver Anova) .

Tabla 1. Comparación de medias de edad de pacientes menores de edad diagnosticados con dengue

\begin{tabular}{llrrr}
\hline & & Valores & IC 95\% & \\
\hline Casos & Media & 4,30 & 1,51 & 7,57 \\
$(\mathrm{~N}=14)$ & Desv. Est. & 5,55 & & \\
Controles & Media & 5,60 & 4,36 & 7,07 \\
$(\mathrm{~N}=56)$ & Desv. Est. & 5,17 & & \\
Total & Media & 5,34 & 4,23 & 6,68 \\
$(\mathrm{~N}=70)$ & Desv. Est. & 5,23 & & \\
& & & F & Sig. \\
\hline ANOVA & & & & \\
\hline Edad Caso- & Inter- & (Combinadas) & ,688 \\
Control & grupos & & & \\
\hline
\end{tabular}

En la tabla 2 se observa la distribución según el sexo de los casos y los controles, no se encontraron diferencias en la distribución del sexo en el grupo de casos, ni en el grupo de controles, ni entre los grupos.

Tabla 2. Distribución por género de pacientes menores de edad diagnosticados con dengue

\begin{tabular}{|c|c|c|c|c|c|c|}
\hline & \multicolumn{3}{|l|}{ Casos } & \multicolumn{2}{|c|}{ Controles } & \multirow[b]{2}{*}{ IC 95\% } \\
\hline & Frecuencia & $\%$ & IC 95\% & Frecuencia & $\%$ & \\
\hline Femenino & 6 & 42,9 & $14,3-64,3$ & 24 & 42,9 & $32,9-54,3$ \\
\hline Masculino & 8 & 57,1 & $35,7-85,7$ & 32 & 57,1 & $45,7-67,1$ \\
\hline Total & 14 & 100,0 & & 56 & 100,0 & \\
\hline
\end{tabular}

En la tabla 3 se observa el área de residencia de los casos y los controles, en los dos grupos los individuos proceden mayoritariamente del área urbana, sin diferencia estadística entre los grupos.
En la tabla 4 se observa el aseguramiento con el que contaban el grupo caso y el grupo control, en los dos grupos predomina el régimen subsidiado, sin presentarse diferencia estadística. 
Tabla 3. Área de residencia de pacientes menores de edad diagnosticados con dengue

\begin{tabular}{|c|c|c|c|c|c|c|}
\hline & Casos & & & ntroles & & \\
\hline & Frecuencia & $\%$ & IC 95\% & Frecuencia & $\%$ & IC 95\% \\
\hline Urbano & 12 & 85,7 & $64,3-100$ & 47 & 83,9 & $82,1-98,2$ \\
\hline Rural & 2 & 14,3 & , 0-35,7 & 5 & 8,9 & $1,8-17,9$ \\
\hline Total & 14 & 100,0 & & 58 & 100,0 & \\
\hline
\end{tabular}

Tabla 4. Tipo de seguridad social de pacientes menores de edad diagnosticados con dengue

\begin{tabular}{lrrrrrr}
\hline & $\begin{array}{c}\text { Casos } \\
\text { Frecuencia }\end{array}$ & \multicolumn{1}{c}{$\begin{array}{c}\text { Controles } \\
\text { F }\end{array}$} & IC 95\% & Frecuencia & \% & \multicolumn{1}{c}{ IC 95\% } \\
\hline Contributivo & 4 & 28,6 & $7,1-57,1$ & 11 & 19,6 \\
Subsidiado & 6 & 42,9 & $14,5-71,4$ & 27 & 48,2 & $35,7-58,6$ \\
No asegurado & 4 & 28,6 & $7,1-57,1$ & 18 & 32,8 \\
Total & 14 & 100,0 & & 58 & 100,0 \\
\hline
\end{tabular}

En la tabla 5 se observa el cálculo de la razón de las ventajas (OR) la cual evidencia que para la mortalidad por dengue se encuentran 17,6 veces más en menores de edad que recibieron Dipirona, con un intervalo de confianza de $95 \%$ entre $3,7 \mathrm{y}$ 84,5. La prueba exacta de Fisher demuestra la significancia estadística para esta medida de riesgo.

Tabla 5. Estimación de riesgo de mortalidad de pacientes menores de edad diagnosticados con dengue

\begin{tabular}{|c|c|c|c|c|c|c|c|}
\hline & \multicolumn{2}{|c|}{$\begin{array}{c}\text { Casos } \\
n=14\end{array}$} & \multicolumn{2}{|c|}{$\begin{array}{c}\text { Controles } \\
n=56\end{array}$} & \multirow{2}{*}{$\begin{array}{c}\text { Razón de ventajas para } \\
\text { uso de Dipirona } \\
\text { OR }\end{array}$} & \multirow{2}{*}{$\begin{array}{l}\text { IC 95\% } \\
\text { Inf - Sup }\end{array}$} & \multirow{2}{*}{$\begin{array}{c}\text { Valor P } \\
\text { Test Fisher }\end{array}$} \\
\hline & $\mathrm{Fr}$ & $\%$ & $\mathbf{F r}$ & $\%$ & & & \\
\hline $\begin{array}{l}\text { Expuestos a } \\
\text { Dipirona }\end{array}$ & 7 & 50 & 3 & 5.5 & 17,66 & $3,7-84,5$ & ,0002 \\
\hline
\end{tabular}

\section{Discusión}

La reducción de la mortalidad por dengue es prioridad para los sistemas de salud, siendo una de las medidas la mejora de la atención clínica ${ }^{2,15}$ y una de las prioridades en investigación para la optimización y estandarización de los procedimientos de atención clínica ${ }^{19}$.

La mortalidad por dengue puede estar relacionada con el virus y el hospedero, como la patogenia viral y la susceptibilidad de la población, pero está aún mayor relacionada con el nivel de preparación del sistema de salud ${ }^{12}$, por eso evitar la trasmisión del dengue es de igual importancia.

Los resultados de este estudio en la comparación del grupo caso y el grupo control muestran que no hay diferencia estadística para la edad, ni para el sexo, y la procedencia es principalmente de la cabecera municipal, seguida del área rural dispersa, y en los dos grupos predomina el contar con aseguramiento al régimen subsidiado, seguido de no contar con aseguramiento, disminuyendo la influencia de variables de confusión. 
Estimando la asociación entre el uso de Dipirona en menores de 16 años con diagnóstico de dengue y la ocurrencia de casos de mortalidad, la razón de las ventajas (OR) fue de 17,6 con un intervalo de confianza de $95 \%$ entre 3,7 - 84,5 y un valor de $\mathrm{P}$ de 0,0002 . La tasa de mortalidad en niños que recibieron Dipirona es mayor que en el grupo de niños que no recibieron Dipirona, aunque no se encontraron publicaciones sobre la relación del uso de Dipirona en niños con dengue y casos de mortalidad en la búsqueda realizada, se evidencian riesgos al usar la Dipirona en este tipo de pacientes en otras investigaciones.

La primera es un ensayo clínico realizado en Bolivia comparando el uso de la Dipirona y el Acetaminofén evidencio una disminución diaria del número de neutrófilos con un punto muy bajo en el día cinco de tratamiento, y aumento entre 1,5 y 3 veces de la transaminasa en los niños que recibieron Dipirona en el tratamiento del dengue; aunque la conclusión de este estudio fue que la Dipirona es una alternativa razonablemente segura de tratamiento de la fiebre en pacientes con dengue con efectos adversos menores sin repercusión clínica de importancia ${ }^{20}$.

La segunda es un estudio de cohortes prospectivo realizado en Colombia concluyó que la administración de Dipirona en los primeros cuatro días de enfermedad aumentó el riesgo de desarrollar dengue hemorrágico ( $\mathrm{RR}=7,29$; IC95\% 1,79-29,34) y presentaron un recuento de plaquetas significativamente menor (diferencia promedio: 40.110 plaquetas $/ \mathrm{mm}^{3}$; IC95\% 1.597 $78,624 / \mathrm{mm}^{3}$, p=0,04), un mayor riesgo de hemorragia y descenso de plaquetas a valores menores de $50.000 \mathrm{~mm}^{3}$ con la consiguiente asociación a dengue hemorrágico ${ }^{21}$.

Es indispensable continuar con la vigilancia del proceso de mortalidad en pacientes con dengue y su relación con el uso de la Dipirona, podrían hacerse más recisiones en diferentes departamentos que cuentan con base de vigilancia en salud pública. Analizar cada caso de mortalidad ocurrido y el tipo de tratamiento analgésico recibido.
La Dipirona es fuente de debate y restricción en otros países del mundo, fue retirada del mercado en Suecia y Estados Unidos, debido a la aparición de casos de agranulocitosis y anemia aplásica asociados a su uso, cuya mortalidad está cercana a $10 \%{ }^{22}$. Se estima que la incidencia en pacientes hispanos está alrededor de 0,38 casos por un millón de habitantes/año ${ }^{18}$. Los efectos de la Dipirona sobre los neutrófilos en pacientes con dengue pueden ser sinérgicos, ya que en el dengue los neutrófilos sufren una disminución ${ }^{23}$.

Además la Dipirona tiene recomendaciones y restricciones para su uso en pacientes con dengue, de acuerdo a la guía clínica del manejo del dengue de la Organización Panamericana de la Salud (OPS) y según lo contemplado en la Guía de Atención Clínica Integral del paciente con dengue, la Dipirona debe ser considerada para manejo exclusivo de segundo y tercer nivel, no se debe administrar por vía intramuscular, ni en pacientes pediátricos, y cuando se utilice se debe informar riesgos $^{24}$, se requiere prescripción médica, ya que puede causar hipotensión y reducción de los granulocitos ${ }^{25}$. Los principales factores de riesgo en el uso de la Dipirona incluyen la duración del tratamiento, la dosis empleada y el uso concomitante de otros medicamentos que generen mielotoxicidad ${ }^{18}$.

\section{Conclusión}

En el grupo de menores de 16 años con dengue que recibieron Dipirona en su tratamiento se asocia con una mayor mortalidad, en la revisión de literatura realizada no se encontraron resultados similares, por ende se debe seguir estudiando el fenómeno para contar con mayor evidencia científica.

Conflicto de intereses: Ninguno declarado por los autores.

\section{Referencias}

1. Martínez-Torres E. Dengue y dengue hemorrágico: aspectos clínicos. Salud pública de México. 2015;37.

2. Periago MR, Guzmán MG. Dengue y dengue hemorrágico en las Américas. Revista Panamericana de Salud Pública. 2007;21(4):187-91. 
3. Teixeira MG, Costa MCN, Coelho G, Barreto ML. Recent shift in age pattern of dengue hemorrhagic fever, Brazil. Emerging infectious diseases. 2008;14(10):1663-4.

4. Hammond SN, Balmaseda A, Perez L, Tellez Y, Saborío SI, Mercado JC, et al. Differences in dengue severity in infants, children, and adults in a 3-year hospital-based study in Nicaragua. The American journal of tropical medicine and hygiene. 2005;73(6):1063-70.

5. Hubert B, Halstead SB. Dengue 1 virus and dengue hemorrhagic fever, French Polynesia, 2001. Emerg Infect Dis. 2009;15(8):1265-70.

6. Halstead SB, Lan NT, Myint TT, Shwe TN, Nisalak A, Kalyanarooj $\mathrm{S}$, et al. Dengue hemorrhagic fever in infants: research opportunities ignored. Emerging infectious diseases. 2002;8(12):1474-9.

7. San Martín JL, Brathwaite O, Zambrano B, Solórzano JO, Bouckenooghe A, Dayan GH, et al. The epidemiology of dengue in the Americas over the last three decades: a worrisome reality. The American journal of tropical medicine and hygiene. 2010;82(1):128-35.

8. Kabilan L, Balasubramanian S, Keshava S, Thenmozhi V, Sekar G, Tewari S, et al. Dengue disease spectrum among infants in the 2001 dengue epidemic in Chennai, Tamil Nadu, India. Journal of clinical microbiology. 2003;41(8):3919-21.

9. Organization WH. Dengue and severe dengue. Fact sheet N 117. Available from:[Last Updated February 2015] Back to cited text. 2012(3).

10. Ganguly A, Malabadi RB, Bhatnagar PK, Tang X, Das D, Loebenberg $\mathrm{R}$, et al. Production and characterization of monospecific and bispecific antibodies against dengue virus NS1 protein. Journal of virological methods. 2015;220:5-12.

11. Campos KB, Amâncio FF, Araújo VEM, Carneiro M. Factors associated with death from dengue in the state of Minas Gerais, Brazil: Historical cohort study. Tropical Medicine \& International Health. 2015;20(2):211-8.

12. Torres EM. La prevención de la mortalidad por dengue: un espacio y un reto para la atención primaria de salud. Rev Panam Salud Pública. 2006;20(1):61.

13. Salgado DM, Panqueba C, Vega M, Garzón M, Castro D, Rodríguez J. Mortalidad por dengue hemorrágico en niños en Colombia: más allá del choque. Infectio. 2008;12(1):247-53.

14. Gutierrez-Lesmes 0 , Martinez C. Indicador sintético para la medición de la necesidad de investigación y gestión ambiental basado en morbimortalidad ocurrida en 2009-2012, Meta, Colombia. revista luna azúl. 2016;42:154-66.

15. Martínez-Torres E, Polanco-Anaya AC, Pleites-Sandoval EB. ¿ Por qué y cómo mueren los niños con dengue? Revista Cubana de Medicina Tropical. 2008;60(1):0-

16. Davrieux M, Gutiérrez S, Marín D, Pier D, Pais T. Agranulocitosis por Dipirona: a propósito de un caso clínico. Archivos de Pediatría del Uruguay. 2007;78(1):35-40.

17. Arcila-Herrera H, Barragán-Padilla S, Borbolla-Escoboza JR, Canto-Solís A, Castañeda-Hernández G, de LeónGonzález M, et al. Consenso de un grupo de expertos mexicanos: Eficacia y seguridad del Metamizol
(Dipirona). Gaceta médica de México. 2004;140(1):99101.

18. Buitrago-González TP, Calderón-Ospina CA, VallejosNarváez Á. Dipirona: ¿ Beneficios subestimados o riesgos sobredimensionados? Revisión de la literatura. Revista Colombiana de Ciencias Químico-Farmacéuticas. 2014;43(1):173-95.

19. Kroeger A, Nathan MB. Dengue: setting the global research agenda. The Lancet. 2007;368(9554):2193-5.

20. Céspedes Lesczinsky M, Patricio Gutiérrez S, Torrico A, Tobías Paz F. Efectos de la administración de Dipirona en niños tratados por dengue con signos de alarma. Revista de la Sociedad Boliviana de Pediatría. 2015;54(3):121-9.

21. Díaz-Quijano FA, Villar-Centeno LÁ, Martínez-Vega RA. Efecto de la administración temprana de Dipirona sobre la gravedad del dengue en una cohorte prospectiva. Enfermedades infecciosas y microbiologia clinica. 2005;23(10):593-7.

22. Montoya GA, Vaca C, Parra MF. Detección de efectos secundarios asociados a la administración de tramadol y Dipirona en un hospital de alta complejidad. Biomédica. 2009;29(3):369-81.

23. Torrelio A. El hemograma como instrumento diagnóstico básico en pediatría. Revista de la Sociedad Boliviana de Pediatría. 2015;50(2).

24. Malagon JN, Padilla JC, Rojas-Alvarez DP. Guía de Atención Clínica Integral del paciente con dengue. Infectio. 2011;15(4):293-301.

25. Almeida AM, de Oliveira ME. A química medicinal, as reações químicas e os efeitos provocados por medicamentos em caso de dengue. Conexão ciência (Online). 2011;6(2):13-20. 